\title{
Long-term changes in thermospheric composition inferred from a spectral analysis of ionospheric F-region data
}

\author{
C. J. Scott ${ }^{1}$, R. Stamper ${ }^{2}$, and H. Rishbeth ${ }^{3, \dagger}$ \\ ${ }^{1}$ University of Reading, Reading, Berkshire, UK \\ ${ }^{2}$ Rutherford Appleton Laboratory, Chilton, Oxfordshire, UK \\ ${ }^{3}$ School of Physics and Astronomy, University of Southampton, Southampton, UK \\ $\dagger$ deceased, 23rd March 2010
}

Correspondence to: C. J. Scott (chris.scott@ reading.ac.uk)

Received: 17 September 2013 - Revised: 6 January 2014 - Accepted: 17 January 2014 - Published: 17 February 2014

\begin{abstract}
A study of ionospheric data recorded at Slough/Chilton, UK, from 1935 to 2012, has revealed longterm changes in the relative strength of the annual and semiannual variability in the ionospheric F2 layer critical frequencies. Comparing these results with data from the southern hemisphere station at Stanley in the Falkland Islands between 1945 and 2012 reveals a trend that appears to be anti-correlated with that at Chilton. The behaviour of $f o \mathrm{~F} 2$ is a function of thermospheric composition and so we argue that the observed long-term changes are driven by composition change. The ionospheric trends share some of their larger features with the trend in the variability of the aa geomagnetic index. Changes to the semi-annual/annual ratio in the Slough/Chilton and Stanley data may therefore be attributable to the variability in geomagnetic activity which controls the average latitudinal extent of the auroral ovals and subsequent thermospheric circulation patterns. Changes in ionospheric composition or thermospheric wind patterns are known to influence the height of the F2 layer at a given location. Long-term changes to the height of the F2 layer have been used to infer an ionospheric response to greenhouse warming. We suggest that our observations may influence such measurements and since the results appear to be dependent on geomagnetic longitude, this could explain why the long-term drifts observed in F2 layer height differ between locations.
\end{abstract}

Keywords. Atmospheric composition and structure (thermosphere composition and chemistry) - ionosphere (ionosphere-atmosphere interactions) - radio science (instruments and techniques)

\section{Introduction}

The UK ionospheric monitoring group has made continuous measurements of the ionosphere from Slough $\left(51.5^{\circ} \mathrm{N}\right.$, $\left.0.6^{\circ} \mathrm{W}\right)$ and Chilton $\left(51.7^{\circ} \mathrm{N}, 1.3^{\circ} \mathrm{W}\right)$ in the UK since 1931 and Stanley in the Falkland Islands $\left(51.9^{\circ} \mathrm{S}, 57.8^{\circ} \mathrm{W}\right)$ since 1945. These data were instrumental in studying the behaviour of the ionosphere, which is now largely understood. At mid-latitudes, the dominant cause of ionisation in the upper atmosphere is the absorption of solar X-ray and Extreme Ultra Violet radiation. This behaviour is modulated by changes to the thermospheric composition either as a result of seasonal changes to thermospheric circulation patterns (Rishbeth, 1998) or in response to auroral heating, e.g. Davis et al. (1997). More recently these long sequences have proved invaluable in identifying long-term changes in the height of the ionospheric F2 layer predicted as a consequence of climate change (Bremer, 1992; Jarvis et al., 1998). Changes to the long-term occurrence of other ionospheric parameters are less well studied, since such statistics are dependent on good data coverage and consistency in both equipment and data scaling.

Data from the Slough/Chilton and Stanley sequences have been manually scaled following the URSI conventions (Piggott and Rawer, 1961). Equipment has inevitably been changed throughout the sequences and such occasions are recorded. While automatic scaling of ionospheric data is becoming more widespread, it is still necessary to scale the parameters manually if a consistent scientific archive of the data is to be maintained. An advantage of the same person scaling the data over an extended time is that unexpected trends 
in some of the parameters become apparent. Routine manual scaling of the Chilton and Stanley data ceased in 2006 but monthly noon median values are still calculated from manual inspection of the data.

\section{Annual and semi-annual variability in the ionosphere}

Ionospheric densities at all heights show a strong solar cycle variation due to the resulting modulation of solar irradiance and geomagnetic activity. For mid-latitude locations in longitude sectors that are near to the geomagnetic pole (such as Slough/Chilton) there is an annual variation in ionisation with ionospheric densities being greatest in the winter. This is due to seasonal composition changes in the thermosphere at F-region altitudes as originally suggested by Rishbeth and Setty (1961) and later modelled by Zou et al. (2000). In the summer, the thermosphere has a greater concentration of molecular species that increase the ionospheric loss rate through charge exchange with atomic ions followed by dissociative recombination of the molecular ions. This results in lower F-region ionospheric densities in the summer months where the proportion of molecular species is relatively high. In the winter months, downwelling of the meridional thermospheric circulation maintains a thermospheric composition that is dominated by atomic species which have a lower loss rate. This seasonal change in composition exceeds the decrease in ion production due to the seasonal change in solar zenith angle over the same period.

Modelling work (Millward et al., 1996) demonstrated that the semi-annual effect can also be explained by composition and zenith angle changes between the seasons. A semiannual variation results when a station is far enough from the magnetic pole that compositional changes between equinox and winter months are relatively small compared with the associated change in solar zenith angle. This is the case for the ionosphere above Stanley in the Falkland Islands where ionospheric densities peak at the equinoxes.

While these are the dominant trends at each location, the relative strength of each does vary. For example, the semiannual variation was prominent at Slough during the solar minimum year 1933-1934 when the first complete year of ionospheric observations was made (Appleton and Naismith, 1935; Rishbeth, 1998). Since both the annual and semi-annual variations can be explained by the relative influence of changes in composition and zenith angle, a study into how the relative magnitude of these effects varies with time at a given location should provide a useful insight into long-term compositional changes. Such a study has been undertaken for $37 \mathrm{yr}$ of ionospheric data recorded at Concepcin (Bravo et al., 2011) who recorded long-term changes in the relative strength of annual and semi-annual variations in the ionosphere and suggested that these could be explained by changes in composition or thermospheric circulation. We present a similar analysis for the long-term ionospheric data sequences at Slough/Chilton and Stanley.
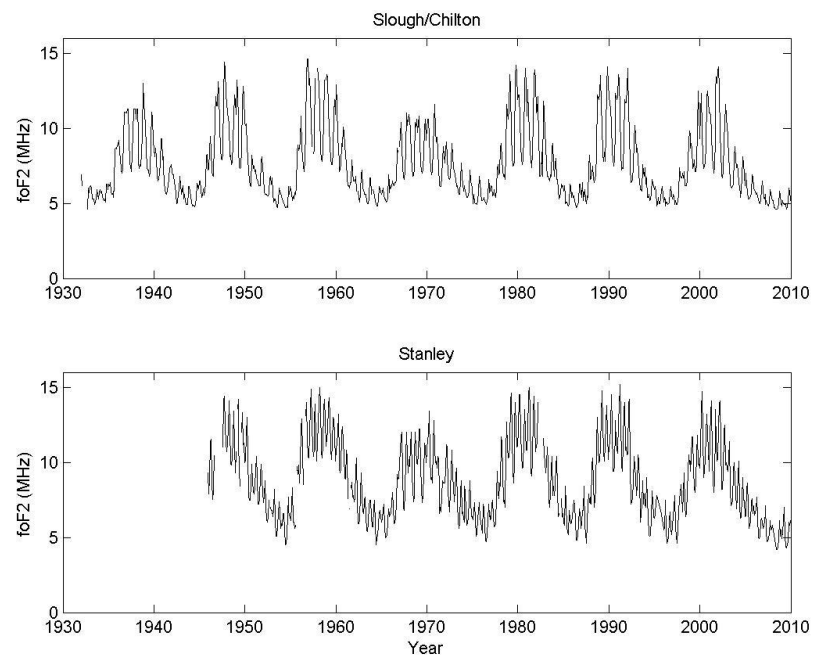

Fig. 1. Long-term variations in monthly median noon $f o \mathrm{~F} 2$ for Slough/Chilton in the UK (top panel) and Stanley in the Falkland Islands (lower panel). The ionosphere above both stations is modulated by the eleven-year solar activity cycle. Superposed with this is a dominant annual variation at Chilton and a semi-annual variation at Stanley.

\section{Long-term changes in the annual and semi-annual components of $f o \mathrm{~F} 2$}

The electron concentration of an ionospheric layer can be determined by probing the ionosphere with short-wave radio pulses. The parameter $f o \mathrm{~F} 2$ represents the highest radio frequency returned from the peak of the F2 layer and is related to the peak electron concentration, $N m \mathrm{~F} 2$, by the formula $f o \mathrm{~F} 2=8.98 \sqrt{ } N m \mathrm{~F} 2$. Noon monthly median $f_{o} \mathrm{~F} 2$ values for Slough/Chilton and Stanley (Fig. 1) were used to investigate long-term changes in the relative magnitude of the annual and semi-annual components. In order to do this, a Lomb-Scargle spectral analysis was carried out on the data using an eleven-year sliding window to select data. Each window of data was detrended by fitting and subtracting a cubic function before carrying out the spectral analysis. This was done to remove any influence of the background trend in the resulting power spectra. A Lomb-Scargle spectral analysis effectively yields a vector of powers attributed to each frequency for the eleven-year window of data considered. Sliding the window in time results in a series of spectral analyses, each tagged with the central time in the time window. The series of spectra is shorter than the original time series by the window length since only complete eleven-year data sets can be used in this analysis. Figures 2 and 3 show the resulting periodograms for local noon at Slough/Chilton and Stanley, respectively. It can be seen that the spectra at both stations contain both annual and semi-annual components with, as expected, the semi-annual component being more significant at Stanley while the annual component dominates the spectra at Slough. 


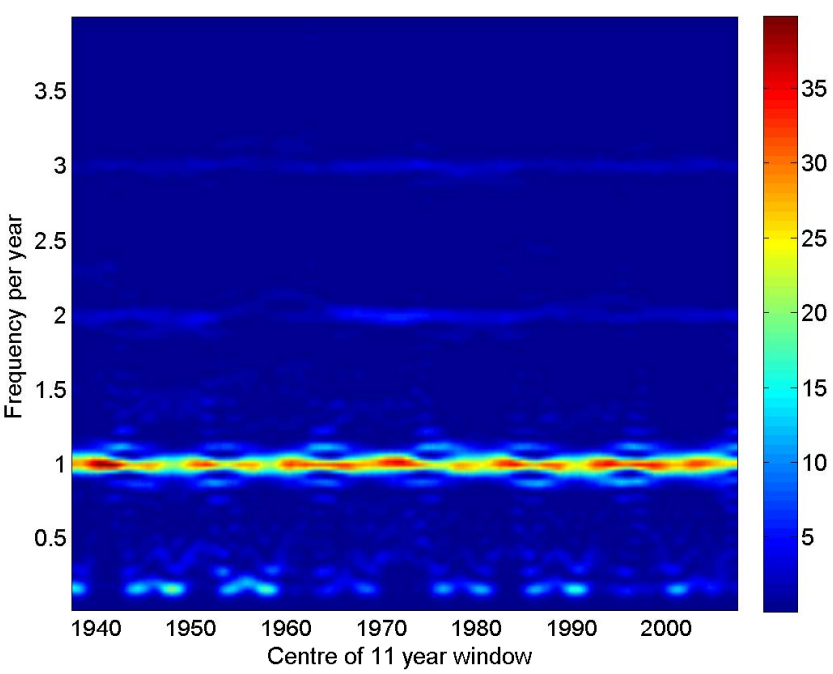

Fig. 2. Lomb-Scargle periodogram for the local noon ionospheric F2 layer above Slough/Chilton. The relative power between frequencies is displayed in the colour axis showing a dominant annual variation (frequency per year of 1).

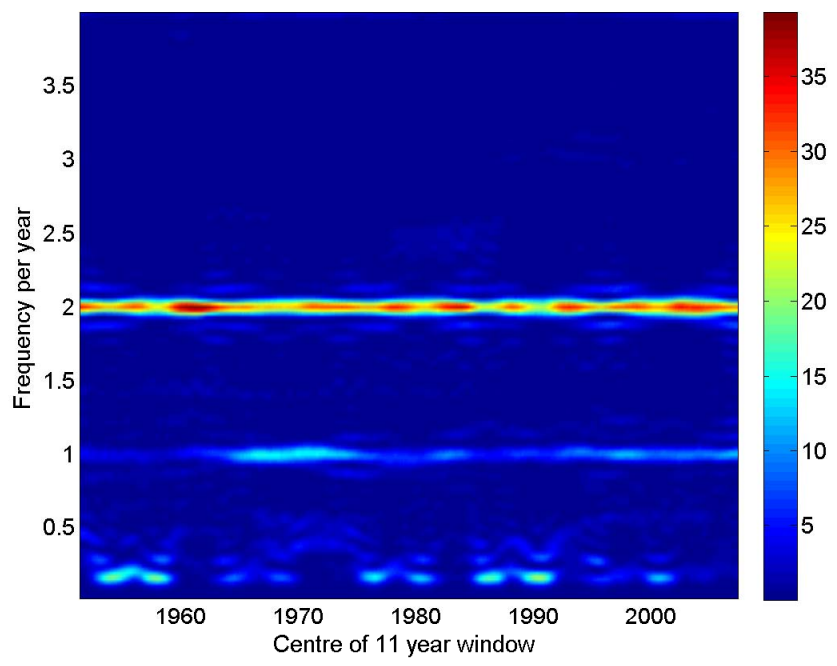

Fig. 3. Lomb-Scargle periodogram for the local noon ionospheric F2 layer above Stanley. The relative power between frequencies is displayed in the colour axis showing a dominant semi-annual variation (frequency per year of 2).

To investigate further, the total power in the range $\pm 0.05 \mathrm{~Hz}$ either side of the annual and semi-annual components was calculated, and the ratio of these powers plotted for each station as a function of time. Since the semi-annual component is not always present, this was used as the numerator when calculating the ratios.

These semi-annual/annual power ratios for the stations are shown in Fig. 4 as a function of time. While changes in the semi-annual/annual power ratio are much larger in the Stanley data sequence than at Slough/Chilton, the trends seen at the two stations contain prominent anti-correlated features.

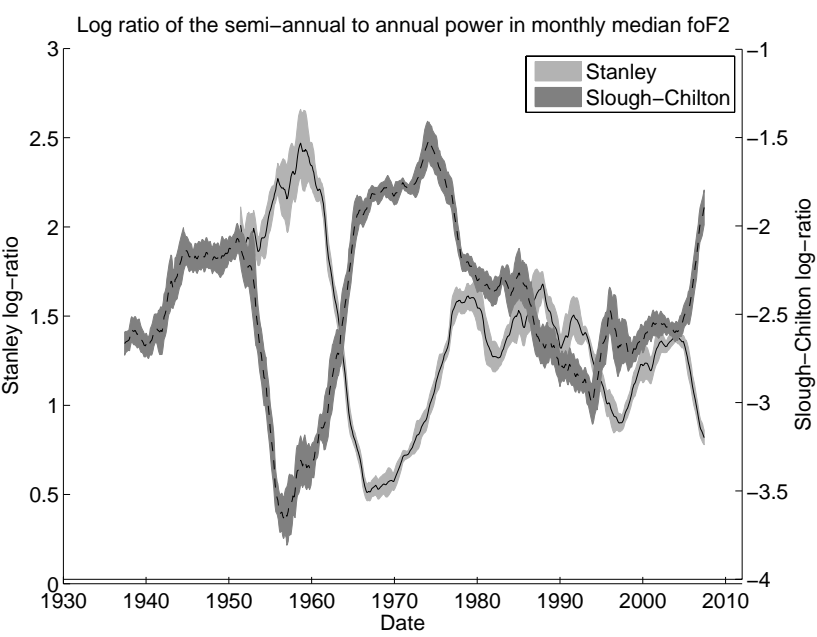

Fig. 4. Time series of the $\log$ ratio of semi-annual/annual values seen at Slough/Chilton (dashed line with dark grey error band) and Stanley (solid line with light grey error band) at local noon. The grey error bands represent the error in the mean over the frequency band used to produce each line.

For comparison with the work of Bravo et al. (2011), who found that the annual variation of the $f o \mathrm{~F} 2$ layer varied between day and night, the analysis was repeated for local midnight at both stations. The resulting semi-annual/annual log power ratios are shown in Fig. 5. It can be seen that the annual component dominates at both stations during the night (the magnitude of the ratio decreases dramatically) and the variability at the two stations no longer anti-correlates.

\section{Discussion and conclusions}

If an ionospheric parameter changes over time, and this is not due to instrumental changes, there must have been some change in ionospheric physics or chemistry. Modulation of the F2 peak could be the result of changes in ion production and loss or thermospheric temperature, composition and winds. Compositional changes cannot be due to thermal expansion or contraction caused by changes in temperature, which does not affect the relative abundances of the gases at a given pressure level (Garriott and Rishbeth, 1963) (provided all gases are diffusively separated with their own scale heights). Some other change must be occurring, therefore, to increase the molecular abundances, probably composition changes at the lower boundary of the diffusively separated region around $100 \mathrm{~km}$. This may provide evidence of a more dynamic thermosphere at the turbopause level, above which the composition is controlled gravitationally.

As described in Sect. 2, peak electron concentration and the related critical frequency $f o \mathrm{~F} 2$ are largely controlled by the neutral composition of the ambient thermosphere, e.g. Zou et al. (2000), so the spectral changes described in Sect. 2 


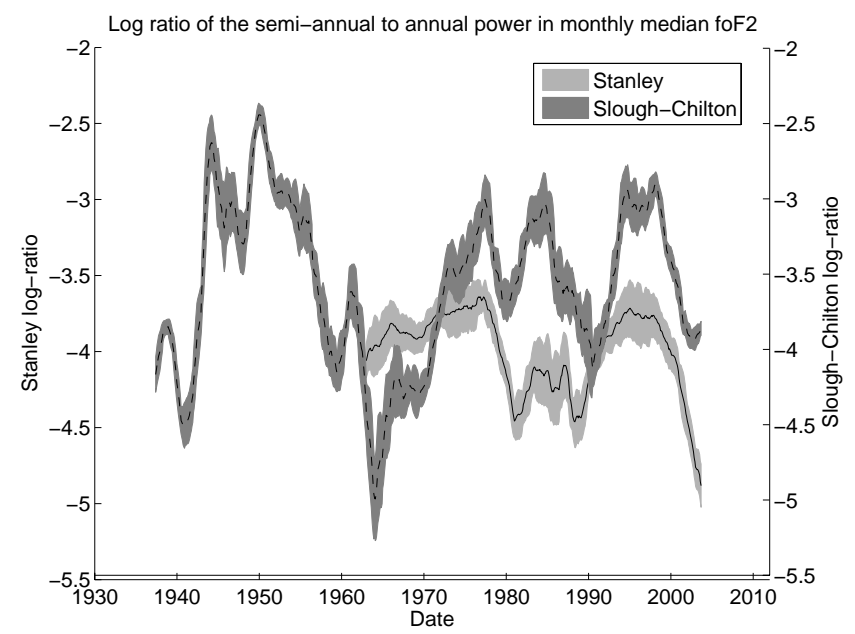

Fig. 5. Time series of the $\log$ ratio of semi-annual/annual values seen at Slough/Chilton (dashed line with dark grey error band) and Stanley (solid line with light grey error band) at local midnight. The grey error bands represent the error in the mean over the frequency band used to produce each line. Note the change in scale compared with Fig. 4.

may well indicate changes in thermospheric composition. The modelling described in Zou et al. (2000) shows that the composition at mid-latitudes varies predominately seasonally (summer/winter) in longitudes near the magnetic pole ("near-pole sectors"), including Slough/Chilton, and semiannually at longitudes remote from the magnetic poles ("farfrom-pole" sectors), including Stanley, as illustrated from worldwide ionosonde data by Torr and Torr (1973).

A clue as to the source of the atmospheric forcing which may contribute to the changes seen in the spectral analysis of $f o \mathrm{~F} 2$ may come from the more striking features in semiannual/annual ratio trends. Slough/Chilton data (Fig. 4) show a large decrease prior to 1960 followed by a rise and subsequent fall (with the opposite occurring for the largest features at Stanley). The semi-annual/annual power ratio observed at Stanley (and the inverse of the same parameter observed at Slough/Chilton) bears a striking resemblance to the variation in geomagnetic activity as determined from the aa index. The aa index along with annual standard deviations in aa are plotted in Fig. 6. These have been smoothed with an eleven-year running mean for comparison with the semi-annual/annual power ratios presented in Figs. 4 and 5.

Variation in geomagnetic activity modulates the mean extent of the auroral oval, which in turn influences the thermospheric circulation that largely controls the chemical composition (Rishbeth, 1998). The UK is likely to be sensitive to such changes, as it is located on the boundary between different regimes of thermospheric convection, as shown by modelling (Zou et al., 2000), while Stanley, in a "far-frompole" longitude, would be closer to the sub-auroral downwelling region as a result of increased auroral activity. Any
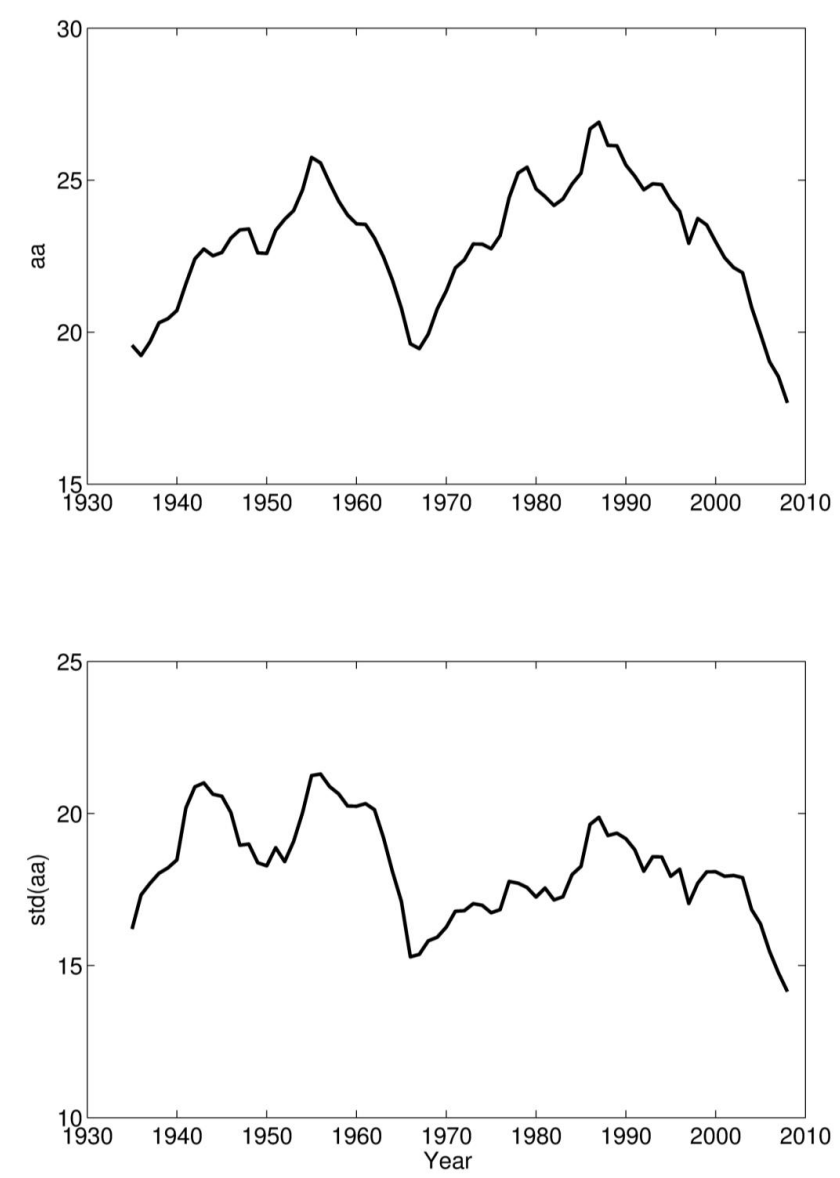

Fig. 6. Time series of annual mean values (top panel) and annual standard deviation (lower panel) of the geomagnetic aa index. For both these parameters, annual values were calculated from daily data and then smoothed with an eleven-year running window for comparison with Figs. 4 and 5.

modulation in the size of the auroral oval would be expected to be mirrored in the conjugate hemisphere. As the ionosphere above Stanley exhibits the opposite trend to that above Slough/Chilton, the cause of the changes must be more complex.

Changes in the semi-annual/annual power ratio are far smaller at Slough/Chilton but their anti-correlation with the measurements at Stanley are striking. One possible argument to explain the observed variations at Chilton could be as follows: Around 1955 the aa index and its variability were relatively high, with enhanced upwelling in the summer hemisphere and the auroral oval more often expanding equatorward towards this "near-pole" location. Enhanced upwelling in the summer hemisphere along with closer proximity to the sub-auroral downwelling region in the winter months would enhance the annual variation in composition, reducing the relative influence of seasonal changes in solar zenith angle thereby weakening any semi-annual component of ionospheric variability. As geomagnetic activity declined 
to a mimimum around 1966 , the auroral oval would have contracted polewards on average, reducing Slough/Chilton's proximity to the auroral downwelling region in the winter months. The difference in thermospheric composition between summer and winter months would likely be reduced under such a scenario, increasing the relative effect of seasonal changes in solar zenith angle resulting in an enhanced semi-annual component of ionospheric variability. During the most recent solar cycle the reduced variation in aa has once again resulted in the semi-annual component increasing in strength relative to the annual variation.

Conversely, the ionosphere above Stanley has followed the opposite trend. With reference to Fig. 4, around 1955, aa values were high and very variable, which would enhance thermospheric upwelling in the summer hemisphere and move the auroral oval equatorward on average, towards Stanley. While this would bring the sub-auroral downwelling region closer to Stanley, it would remain further from the sub-auroral downwelling region than Slough/Chilton due to its location on a "far-from-pole" longitude. If the increased molecular composition resulting from an enhanced upwelling in the summer hemisphere dominated over the composition changes resulting from the modulation in proximity of the sub-auroral downwelling region, a molecularrich environment would be enhanced throughout the year, thereby increasing the relative influence of seasonal changes in solar zenith angle and subsequently the amplitude of the semi-annual effect in ionospheric variability.

Around 1966-1970, the aa decreased in value and became less variable, reducing thermospheric upwelling in the summer hemisphere and causing the auroral ovals to contract polewards. If the annual variation in composition caused by the reduction in thermospheric upwelling in the summer hemisphere were to dominate over the reduced compositional change caused by the poleward retreat of the sub-auroral downwelling region in the winter hemisphere then the annual variation in composition would be enhanced relative to seasonal changes in solar zenith angle, decreasing the relative power of the semi-annual ionospheric variation. As geomagnetic activity increased throughout the period from 19661990 the semi-annual effect increased in amplitude. In the most recent and unusually quiet solar cycle, the magnitude and variability of geomagnetic activity has once again declined, thermospheric upwelling will have reduced and the auroral ovals have, on average, retreated polewards, causing the semi-annual effect to become less prominent at Stanley.

At local midnight thermospheric wind fields will differ from daytime circulation patterns and it is to be expected that any solar zenith angle effect would be significantly reduced. While the long-term variations at the two stations are dominated by the annual component at night, these sequences also contain variations that are seen in the aa data, in particular the prominent dip in the mid-1960s.
The above descriptions are extremely qualitative since a rigorous investigation into the observed long-term changes in $f o \mathrm{~F} 2$ behaviour would involve detailed modelling not only of the thermospheric composition but also of the associated changes to thermospheric wind fields. Meridional thermospheric winds are known to modulate both the height and density of the F2 peak by moving ionisation along inclined magnetic field lines. Altering the altitude of the ionisation in this way in turn affects the loss rate, modulating the F2 peak density (Rishbeth, 1998).

The drift of the magnetic poles during the period of these observations is another potential factor that could contribute to the difference between the two stations in the annual/semiannual behaviour of $f o \mathrm{~F} 2$. The seasonal variation of $f o \mathrm{~F} 2$ depends on geomagnetic location. According to the International Geomagnetic Reference Field Geomagnetic Field Model, between 1930 and 2010, Slough/Chilton became further from the north magnetic pole (the corrected geomagnetic, CGM, latitude changed from $50.17^{\circ} \mathrm{N}$ to $48.1^{\circ} \mathrm{N}$ ) and Stanley became closer to the south magnetic pole (the CGM latitude changed from $34.92^{\circ}$ to $39.21^{\circ} \mathrm{S}$ ). In both cases the drift was smooth and in itself does not explain the variations seen in $f o \mathrm{~F} 2$ behaviour. In principle, the drift of the poles may modulate geomagnetic activity, but that effect is minimal at the longitudes of the UK and Australian stations used to compile the aa index (Clilverd et al., 1998). At Stanley the effect may be more significant, and could contribute to the behavioural changes seen in $f o F 2$. If a difference in sensitivity to the changing position of the magnetic poles and auroral ovals does exist, it may contribute to the anti-correlation in long-term trends between the two stations.

To test if proximity to the magnetic pole is indeed influencing the relative magnitude of the annual and semi-annual variability, the analysis was repeated for the (much shorter) data sequence from the ionospheric station located on the Argentine Islands between 1957 and 1988. These results (not shown) correlate well with the Stanley noon data trend but with much larger values (stronger semi-annual component), the $\log$ ratio increasing from around 1.2 in the mid-1960s to a peak of around 3.4 in the mid-1970s. At night the ratios are once again dominated by the annual component with significantly reduced $\log$ ratios ranging in value from -4.5 in the mid-1960s to a peak of around -3.6 in the mid-1970s. Since the Argentine Islands are at a similar geomagnetic longitude to Stanley (longitude $9.9^{\circ}$ East) and are closer to the magnetic pole (latitude $50.9^{\circ}$ South) this variability agrees with the hypothesis that the long-term variability is being modulated by geomagnetic activity.

Roble and Dickinson (1989) investigated the influence on the upper atmosphere of increased tropospheric $\mathrm{CO}_{2}$ concentrations. Through modelling work they concluded that the thermosphere would cool by around $50 \mathrm{~K}$ as a result. Rishbeth (1990) further speculated that the height of the ionospheric F2 region, $h m \mathrm{~F} 2$, would be reduced by several kilometres in such a scenario, a conclusion verified 
by subsequent modelling work (Rishbeth and Roble, 1992). Since then there has been much interest in determining whether it is possible to detect any long-term decrease in the height of the ionosphere, a challenging measurement to make given the sensitivity of the ionosphere to solar and geomagnetic activity. Bremer $(1992,1998)$ studied the longterm variability of ionospheric stations across Europe with stations demonstrating a variety of trends. Jarvis et al. (1998) presented a significant decrease in F2 layer height (around $20 \mathrm{~km}$ ) over Stanley and concluded that while this was consistent with the anticipated response to greenhouse warming, it could also result from changes to the thermospheric wind pattern. Bremer (2001) also inferred thermospheric changes from ionospheric data recorded at various locations and concluded that the scatter of trends observed in the long-term behaviour of ionospheric F-region height made it impossible to infer any long-term trends due to greenhouse warming.

Changes to thermospheric circulation can influence the height of the ionospheric F2 layer through the meridional component of the neutral wind driving ions and electrons along geomagnetic field lines that are inclined to the horizontal with a dip angle, $I$. For a horizontal wind with a meridional speed, $U$, the vertical component, $W$, is $U \sin I \cos I$ (Rishbeth, 1998). An upward drift raises the peak and increases the peak density, $N m \mathrm{~F} 2$, according to the ratio $q / \beta$ at the increased altitude (where $q$ and $\beta$ are the ion production and loss rates, respectively).

Millward et al. (1996) investigated the effects of composition and meridional wind fields on the formation of the semiannual variation in $f o F 2$. They used a coupled thermosphereionosphere-magnetosphere model to investigate the yearly variations at two Southern Hemisphere sites with similar geographic latitudes but with very different magnetic latitudes. Their results show that at the location on a far-from-pole longitude, the mean molecular mass of the thermosphere was higher and less varied than for the station nearer the geomagnetic pole (their Fig. 3). This coincided with an $h m \mathrm{~F} 2$ some $40 \mathrm{~km}$ lower at the far-from-pole location than that near-pole location (their Fig. 4). A comparison of the meridional winds showed that the poleward wind at the far-frompole station was consistently around $75 \mathrm{~km} \mathrm{~s}^{-1}$ greater than the same wind component at the near-pole location. From these results it can be concluded that proximity to the auroral downwelling region modulates both the composition and the meridional wind field at a fixed geographic location, modulating both the height and density of the F2 layer.

Any long-term changes in the thermospheric circulation and/or composition will similarly influence the long-term density and height of the F2 layer. Furthermore, as these trends are not expected to be the same at all locations (as modelled by Millward et al., 1996 and shown in this paper for the ionosphere above Chilton and Stanley) they may contribute towards the observed longitudinal variation in $h m \mathrm{~F} 2$, enhancing or obscuring any trends generated in response to greenhouse warming.
Assessing the composition and circulation changes necessary to account for the observed changes in annual/semiannual behaviour of $f o \mathrm{~F} 2$ will involve detailed modelling but determining the impact of such changes at a variety of geographic locations will enable the subsequent impact on the height of the F2 layer to be estimated. Only then will it be possible to model how these inferred compositional changes will influence the long-term behaviour of the height of the ionospheric F2 layer.

Acknowledgements. The authors thank M. Lockwood and M. Jarvis for their time and useful suggestions and Natalia Papitashvili of NASA/GSSF for providing the CGM coordinates program via http://omniweb.gsfc.nasa.gov/vitmo/cgm_vitmo.html. The authors would also like to thank their referees for their extremely helpful evaluation of the original manuscript. Henry Rishbeth provided much of the motivation for this work, was an enthusiastic contributor to early discussions and co-wrote the initial manuscript. He was an inspirational mentor and we dedicate this paper to him.

Topical Editor Keisuke Hosokawa thanks A. J. Foppiano and G. Millward for their help in evaluating this paper.

\section{References}

Appleton, E. V. and Naismith, R.: Some further measurements of upper atmosphere ionisation, Proc. Roy. Soc., A150, 685-708, 1935.

Bravo, M. A., Foppiano, J., and Abarca del Rio, R.: Long-Term Dependencies of Annual and Semiannual Components of $\mathrm{NmF} 2$ over Concepcin, The Open Atmos. Sci. J., 5, 2-8, 2011.

Bremer, J.: Ionospheric trends in mid-latitudes as a possible indicator of the atmospheric greenhouse effect, J. Atmos. Terrest. Phys, 54, 1505-, 1992.

Bremer, J.: Trends in the ionospheric $\mathrm{E}$ and $\mathrm{F}$ regions over Europe, Ann. Geophys., 16, 986-996, doi:10.1007/s00585-998-0986-9, 1998.

Bremer, J.: Trends in the thermosphere derived from global ionosonde observations, Adv. Space Res., 28, 997-1006, 2001.

Clilverd, M. A., Clark, T. D. G., Clarke, E., and Rishbeth, H.: Increased magnetic storm activity from 1868 to 1995, J. Atmos. Solar-Terr. Phys., 60, 1047-1056, 1998.

Davis, C. J., Wild, M. N., Lockwood, M., and Tulunay, Y. K.: Ionospheric and geomagnetic responses to changes in IMF $B_{Z}$ : a superposed epoch study, Ann. Geophys., 15, 217-230, doi:10.1007/s00585-997-0217-9, 1997.

Garriott, O. K. and Rishbeth, H.: Effects of temperature changes on the electron density profile in the F2 layer, Planet. Space Sci., 11, 587-590, 1963.

Jarvis, M. J., Jenkins, B., and Rodger, G. A.: Southern hemisphere observations of a long-term decrease in $\mathrm{F}$ region altitude and thermospheric wind providing possible evidence for global thermospheric cooling, J. Geophys. Res., 103, 20774-20787, 1998.

Millward, G. H., Moffet, R. J., Quegan, S., Fuller-Rowell, T. J.: Ionospheric F2 layer seasonal and semi-annual variations, J. Geophys. Res., 101, 5149-5156, 1996.

Piggott, W. R. and Rawer, K.: URSI Handbook of Ionogram Reduction, Elsevier, New York, 1961. 
Rishbeth, H.: A greenhouse effect in the ionosphere?, Planet. Space Sci., 38, 945-948, 1990.

Rishbeth, H.: How the thermospheric circulation affects the ionospheric F2-layer, J. Atmos. Solar-Terr. Phys., 60, 1385-1402, 1998.

Rishbeth, H. and Roble, R. G.: Cooling of the upper atmosphere by enhanced greenhouse gases - Modelling of the thermospheric and ionospheric effects, Planet. Space Sci., 40, 1011-1026, 1992.

Rishbeth, H. and Setty, C. S. G. K.: The F-layer at sunrise, J. Atmos. Terr. Phys., 21, 263-276, 1961.
Roble, R. G. and Dickinson, R. E.: How will changes of carbon dioxide and methane modify the mean structure of the mesosphere and thermosphere?, Geophys. Res. Lett., 16, 1441-1444, 1989.

Torr, M. R., and Torr, D. G.: The seasonal behaviour of the F2-layer of the ionosphere, J. Atmos. Terrest. Phys., 35, 2237-2251, 1973.

Zou, L., Rishbeth, H., Müller-Wodarg, I. C. F., Aylward, A. D., Millward, G. H., Fuller-Rowell, T. J., Idenden, D. W., and Moffett, R. J.: Annual and semiannual variations in the ionospheric F2-layer. I. Modelling, Ann. Geophys., 18, 927-944, doi:10.1007/s00585000-0927-8, 2000. 